\title{
A Comparison of Annealing Techniques for Academic Course Scheduling
}

\author{
M. A. Saleh Elmohamed ${ }^{1}$, Paul Coddington ${ }^{2}$, and Geoffrey Fox ${ }^{1}$ \\ 1 Northeast Parallel Architectures Center \\ Syracuse University, Syracuse, NY 13244, USA \\ \{saleh, gcf $\}$ @npac.syr.edu \\ 2 Department of Computer Science \\ University of Adelaide, S.A. 5005, Australia \\ paulc@cs.adelaide.edu.au
}

\begin{abstract}
In this study we have tackled the NP-hard problem of academic class scheduling (or timetabling) at the university level. We have investigated a variety of approaches based on simulated annealing, including mean-field annealing, simulated annealing with three different cooling schedules, and the use of a rule-based preprocessor to provide a good initial solution for annealing. The best results were obtained using simulated annealing with adaptive cooling and reheating as a function of cost, and a rule-based preprocessor. This approach enabled us to obtain valid schedules for the timetabling problem for a large university, using a complex cost function that includes student preferences. None of the other methods were able to provide a complete valid schedule.
\end{abstract}

\section{Introduction}

The primary objective of this study is to derive an approximate solution to the problem of university class scheduling, or timetabling, which can be summarized as follows: given data sets of classes and their days, enrollments, and instructors; rooms and their capacities, types, and locations; distances between buildings; priorities of each building for different departments; and students and their class preferences; the problem is to construct a feasible class schedule satisfying all the hard constraints and minimizing the medium and soft constraints. Hard constraints are space and time constraints that must be satisfied, such as scheduling only one class at a time for any teacher, student, or classroom. Medium and soft constraints are student and teacher preferences that should be satisfied if possible.

The timetabling problem (TTP) is a high-dimensional, non-Euclidean, multiconstraint combinatorial optimization problem, and is consequently very difficult to solve. This problem has been tackled by many researchers, mostly in the field of operations research. A number of different heuristics have been tried on different instances of the problem, from high school to university course scheduling (see the reviews by de Werra [5] and Shaerf [30] and the papers collected in Ref. [4]). For small to medium size problems, such as exam scheduling, high 
school scheduling, or course scheduling for a university department, many of these methods work well. However no particular method has yet been shown to produce good results for real-world problems on a much larger scale, such as scheduling all courses for a large university, which we address in this paper. Also, we are not aware of any large scale study that takes into account constraints due to student preferences, as we have done.

We have used data for classes at Syracuse University. Currently this problem is handled by the university scheduling department in a semi-automated fashion. A scheduling program is used to find a partial solution, and substantial manual effort is required to iterate towards a final solution. Also, when scheduling a certain semester (e.g. fall 1996), a template of a previous semester (e.g. fall $1995)$ is used as part of the input data.

We have applied the following optimization techniques to this problem:

1. A rule-based expert system.

2. Mean-field annealing.

3. Simulated annealing with geometric cooling.

4. Simulated annealing with adaptive cooling.

5. Simulated annealing with adaptive cooling and reheating as a function of cost.

6. Simulated annealing (using each of the three different cooling schedules) with a rule-based preprocessor to provide a good initial solution.

The best results were obtained using simulated annealing with adaptive cooling and reheating as a function of cost, and with a rule-based preprocessor to provide a good initial solution. Using this method, and with careful selection of parameters and update moves, we were able to generate solutions to the class scheduling problem using real data for a large university. None of the other methods were able to provide a complete valid solution.

\section{The Timetabling Problem}

Timetabling is the assignment of time slots to a set of events, subject to constraints on these assignments. The NP-complete professors and classes timetabling problem $[7,13,14]$ is a constraint satisfaction problem that can be briefly stated as follows:

For a certain school with $N_{p}$ professors, $N_{q}$ classes, $N_{x}$ classrooms and lecture halls, and $N_{s}$ students, it is required to schedule $N_{l}$ professor-class pairs within a time limit of $N_{t}$ time slots producing a legal schedule. A legal schedule needs to be found such that no professor, class, or student is in more than one place at a time, and no room is expected to accommodate more than one lesson at a time or more students than its capacity.

The constraints for this problem can be hard, medium or soft. The medium and soft constraints have an associated cost (or penalty), and if they are not satisfied, the goal is to minimize this cost. Soft constraints have a lower priority (and thus lower cost) than medium constraints. The hard constraints must be 
satisfied, so their associated cost must be reduced to zero. A feasible schedule is one that satisfies all the hard constraints.

Hard constraints are usually constraints that physically cannot be violated. This includes events that must not overlap in time, such as:

- classes taught by the same professor,

- classes held in the same room,

- a class and a recitation or a lab of the same class.

Another examples are space or room constraints:

- A class cannot be assigned to a particular room unless the capacity of the room is greater than or equal to the class enrollment.

- Some classes, such as laboratories, require a certain type of room.

Medium constraints are usually considered to be those constraints that fall into the gray area between the hard and soft constraints [9]. In our implementation, we define medium constraints to be constraints such as time and space conflicts which, like hard constraints, cannot physically be violated (for example, it is not possible for one person to be in two different classes at the same time). However we consider these constraints to be medium rather than hard if they can be avoided by making adjustments to the specification of the problem. The primary example is student preferences. We cannot expect to be able to satisfy all student class preferences, in some cases, certain students will have to adjust their preferences since certain classes will clash, or will be oversubscribed.

Medium constraints have a high penalty attached to them, although not as high as that associated with the hard constraints. In the final schedule the penalty of these constraints should be minimized and preferably reduced to zero. Some examples of medium constraints are:

- Avoid time conflicts for classes with students in common.

- Eligibility criteria for the class must be met.

- Do not enroll athletes in classes that conflict with their sport practice time (of course, depending on the sport).

Soft constraints are preferences that do not deal with time conflicts, and have a lower penalty (or cost) associated with them. We aim to minimize the cost, but do not expect to be able to reduce it to zero. Some examples are:

- For each student, balance the three-day (Mon, Wed, Fri) as well as the twoday (Tue, Thu) schedules.

- Balance or spread out the lectures over the week.

- Classes may request contiguous time slots.

- Balance enrollment in multi-section classes.

- Lunch and other break times may be specified.

- Professors may request periods in which their classes are not taught.

- Professors may have preferences for specific rooms or types of rooms.

- Minimize the distance between the room where the class is assigned and the building housing its home department. 
Some soft constraints may have higher priority (and thus higher cost) than others. For example, preferences involving teachers will have higher priority than the preferences of students.

The cost function measures the quality of the current schedule and generally involves the weighted sum of penalties associated with different types of constraint violations. The aim of the optimization technique is to minimize the cost function.

\section{Mean-Field Annealing}

One of the potential drawbacks of using simulated annealing for hard optimization problems is that finding a good solution can often take an unacceptably long time. Mean-field annealing (MFA) attempts to avoid this problem by using a deterministic approximation to simulated annealing, by attempting to average over the statistics of the annealing process. The result is improved execution speed at the expense of solution quality. Although not strictly a continuous descent technique, MFA is closely related to the Hopfield neural network [15,17].

Mean-field annealing has been successfully applied to high school class scheduling [14]. For scheduling, it is advantageous to use a Potts neural encoding to specify discrete neural variables (or neurons) for the problem. This is defined in its simplest form as a mapping of events onto space-time slots, for example an event $i$, in this case a professor-class pair $(p, q)$, is mapped onto a space-time slot $a$, in this case a classroom-timeslot pair $(x, t)$. Now, the Potts neurons $S_{i a}$ are defined to be 1 if event $i$ takes place in space-time slot $a$, and 0 otherwise. In this way, the constraints involved can be embedded in the neural net in terms of the weights $w_{i, j}$ of the neural network, which encode a Potts normalization condition such as $\sum_{a} S_{i a}=1$.

For a full derivation of the mean-field annealing algorithm from its roots in statistical physics, see Hertz et al. [15] or Peterson et al. [29]. Here we will just give a brief overview of the method. The basic idea is that it is possible to approximate the actual cost or energy function $E$, which is a function of discrete neural variables $S_{i a}$, by an effective energy function $E^{\prime}$ that can be represented in terms of continuous variables $U_{i a}$ and $V_{i a}$. These are known as mean field variables, since $V_{i a}$ is an approximation to the average value of $S_{i a}$ at a given temperature $T$.

This approach effectively smooths out the energy function and makes it easier to find the minimum value, which is obtained by solving the saddle point equations $\frac{\partial E^{\prime}}{\partial V_{i a}}=0$ and $\frac{\partial E^{\prime}}{\partial U_{i a}}=0$, which generate a set of self-consistent mean field theory (MFT) equations in terms of the mean field variables $U$ and $V$ :

$$
\begin{aligned}
U_{i a} & =-\frac{1}{T} \frac{\partial E}{\partial V_{i a}} \\
V_{i a} & =\frac{e^{U_{i a}}}{\sum_{b} e^{U_{i b}}} .
\end{aligned}
$$


The MFA algorithm involves solving equations 1 and 2 at a series of progressively lower temperatures $T$ : this process is known as temperature annealing. The critical temperature $T_{c}$, which sets the scale of $T$, is estimated by expanding equation 2 around the trivial fixed-point $[13,14] V_{i a}^{(0)}=\frac{1}{N_{a}}$, where $N_{a}$ is the number of possible states of each of the network neurons. For example, for the events defined by professor-class pairs $(p, q)$ mapped onto classroom-timeslots $(x, t)$, we have $N_{p} N_{q}$ neurons, each of which has $N_{x} N_{t}$ possible states, in which case $V_{p q ; x t}^{(0)}=\frac{1}{N_{x} N_{t}}$.

Equations 1 and 2 can be solved iteratively using either synchronous or serial updating. The iterative dynamics to evolve the mean field variables toward a selfconsistent solution is explained in detail by Peterson et al. [28]. The solutions correspond to stable states of the Hopfield network [17]. Observe from equation 2 that any solution to the MF equations respects a continuous version of the Potts condition

$$
\sum_{a} V_{i a}=1 \quad \forall i
$$

\subsection{The Mean-Field Annealing Algorithm}

The generic MFA algorithm appears in Figure 1. At high temperatures $T$, the mean-field solutions will be states near the fixed-point symmetrical maximum entropy state $V_{i a}=1 / N_{a}$. At low temperatures, finding a mean-field solution will be equivalent to using the Hopfield model, which is highly sensitive to the initial conditions and known to be ineffective for hard problems [17]. MFA improves over the Hopfield model by using annealing to slowly decrease the temperature in order to sidestep these problems.

These characteristics are similar to those of simulated annealing, which is no surprise since both it and the mean-field method compute thermal averages over Gibbs distributions of discrete states, the former stochastically and the latter through a deterministic approximation. It is therefore natural to couple the mean-field method with the concept of annealing from high to low temperatures.

In addition to the structure of the energy function, there are three major interdependent issues which arise in completely specifying a mean-field annealing algorithm for a timetabling problem:

- The values of the coefficients of terms in the energy function.

- The types of dynamics used to find solutions of the MFT equations at each $T$.

- The annealing schedule details, i.e. the initial temperature $T(0)$, the rules for deciding when to reduce $T$ and by how much, and the termination criteria.

Peterson et al. [14] introduced a quantity called saturation, $\Sigma$, defined as

$$
\Sigma=\frac{1}{N_{i}} \sum_{i a} V_{i a}^{2},
$$


where $N_{i}$ is the number of events (in this case the number of professor-class pairs). This characterizes the degree of clustering of events in time and/or space, $\Sigma_{\min }=\frac{1}{N_{a}}$ corresponds to high temperature, whereas $\Sigma_{\max }=1$ means that all the $V_{i a}$ have converged to 0 or 1 values, indicating that each event has been assigned to a space-time slot.

1. Choose a problem and encode the constraints into weights $\left\{w_{i j}\right\}$.

2. Find the approximate phase transition temperature by linearizing equation (2).

3. Add a self-coupling $\beta$-term if necessary. In a neural net, this corresponds to a feedback connection from a neuron to itself.

4. Initialize the neurons $V_{i a}$ to high temperature values $\frac{1}{N_{a}}$ plus a small random term such as $\operatorname{rand}[-1,1] \times 0.001 ;$ and set $T(0)=T_{c}$.

5. Until $(\Sigma \geq 0.99)$ do:

- At each $T(n)$, update all $U_{i a}$ and $V_{i a}$ by iterating to a solution of the mean field equations.

$-T(n+1)=\alpha T(n)$, we chose $\alpha=0.9$

6 . The discrete values $S_{i a}$ that specify the schedule are obtained by rounding the mean field values $V_{i a}$ to the nearest integer ( 0 or 1$)$.

7. Perform greedy heuristics if needed to account for possible imbalances or rule violations.

Fig. 1. The Generic Mean-Field Annealing Algorithm

The first step of Figure 1 is to map the constraints of the problem into the neural net connection weights. In our implementation, at each $T_{n}$ the MFA algorithm (Figure 1) performs one update per neural variable (defined as one sweep) with sequential updating using equations 1 and 2. After reaching a saturation value close to 1 (we chose $\Sigma=0.99$ ) we check whether the obtained solutions are valid, i.e. $E_{\text {hard }}=0$. If this is not the case the network is re-initialized and is allowed to resettle. We repeat this procedure a number of times until the best solution is found. A similar procedure was carried out on high school scheduling by Peterson et al. [14].

The MFA implementation was a little more complicated than the implementation of simulated annealing and the expert system, since it had many more parameters to handle, and it was often more difficult to find optimal values for these parameters. For example, one complication is the computation of the critical temperature $T_{c}$, which involved an iterative procedure of a linearized dynamic system. On the other hand, we observed that the convergence time was indeed much less than any of the convergence times of the simulated annealing using the three annealing schedules studied. For more details on our MFA implementation, see Ref. [10]. 


\section{The Rule-Based System}

We have implemented a fairly complex rule-based expert system for solving the timetabling problem, for three reasons. Firstly, it gives us a benchmark as to how well other methods do in comparison to this standard technique. Secondly, a simplified version of the rule-based system is used to provide sensible choices for moves in the simulated annealing algorithm, rather than choosing swaps completely at random, and this greatly improves the proportion of moves that are accepted. Thirdly, we have used this system as a preprocessor for simulated annealing, in order to provide a good initial solution.

Simulated annealing is a very time-consuming, computationally intensive procedure. Using an expert system as a preprocessor is a way of quickly providing a good starting point for the annealing algorithm, which reduces the time taken in the annealing procedure, and improves the quality of the result. Our results clearly support this rationale for the case of academic scheduling.

The rule-based expert system consists of a number of rules (or heuristics) and conventional recursion to assist in carrying out class assignments. We have developed this system specifically for the problem of academic scheduling. The basic data structures or components of the system are:

1. Distance matrix of values between each academic department and every other building under use for scheduling.

2. Class data structure of each class scheduled anywhere in campus. These structures are capable of linking with each other.

3. Room data structure of each room (regardless of type) involved in the scheduling process. Like classes, room structures are also linked with each other.

4. Data structures for time periods to keep track of which hour or time slot was occupied and which was not.

5. Department inclusion data structure giving department inclusion within other larger departments or colleges.

6. Students structures indicating classes of various degree of requirements and preferences for each student.

The basic function of the system is as follows: given data files of classes, rooms and buildings, department-to-building distance matrix, students data, and the inclusion data, using the abovementioned data structures, the system builds an internal database which in turn is used in carrying out the scheduling process. This process involves a number of essential sub-processes such as checking the distances between buildings, checking building, room type and hours occupied, checking and comparing time slots for any conflicts, checking rooms for any space conflict, and keeping track of and updating the hours already scheduled.

The rule-based system uses an iterative approach. The basic procedure for each iteration is as follows. The scheduling of classes is done by department, so each iteration consists of a loop over all departments. The departments are chosen in order of size, with those having the most classes being scheduled first. The system first loops over all the currently unscheduled classes, and attempts to assign them to the first unoccupied room and timeslot that satisfies all the 
rules governing the constraints. Since constraints involving capacity of rooms are very difficult to satisfy, larger classes are scheduled first, to try to avoid not having large enough rooms later for those class sections with large enrollments.

In some cases the only rooms and timeslots that satisfy all the rules will already be occupied by previously scheduled classes. In that case, the system attempts to move one of these classes into a free room and timeslot, to allow the unscheduled class to be scheduled.

Next, the system searches through all the scheduled classes, and selects those that have a high cost, by checking the medium and soft constraints such as how closely the room size matches the class size, how many students have time conflicts, whether the class is in a preferred time period or a preferred building, and so on. Selecting threshold values for defining what is considered a "high" cost in each case is a subjective procedure, but it is straightforward to choose reasonable values. When a poorly scheduled class is identified, the system searches for a class to swap it with, so that the hard constraints are still satisfied, but the overall cost of the medium and soft constraints is reduced.

This process of swapping rooms continues provided all the rules are satisfied and no "cycling" (swapping of the same classes) occurs. Once all the departments have been considered, this completes one iteration. The system continues to follow this iterative procedure until a complete iteration produces no changes to the schedule.

There are many rules dealing with space and hours, type of room, and priority of room. Many are quite complex, but some of the basic rules, such as those implementing the hard constraints, can be quite straightforward - for example, the following is the basic rule for dealing with time and space conflicts for a room:

IF [room(capacity) > class(space-requested)] and [no time conflict in this room] THEN assign the room to the class.

When the rule-based system is used as a preprocessor, it produces a partial schedule as an output, since it is usually unable to assign all of the given classes to rooms and times slots. The output is divided into two parts: the first consists of classes, with their associated professors and students, assigned to various rooms; and the second is a list of classes that could not be assigned due to constraint conflicts.

\section{Simulated Annealing}

Simulated annealing (SA) has been widely used for tackling different combinatorial optimization problems, particularly academic scheduling $[35,7,8]$. The basic algorithm is described in Figure 2. The results obtained depend heavily on the cooling schedule used. We initially used the most commonly known and used schedule, which is the geometric cooling, but later tried adaptive cooling, as well as the method of geometric reheating based on cost [3]. 
A comprehensive discussion of the theoretical and practical details of SA is given in $[1,27,32,34]$. It suffices here to say that the elementary operation in the Metropolis method for a combinatorial problem such as scheduling is the generation of some new candidate configuration, which is then automatically accepted if it lowers the cost $(C)$, or accepted with probability $\exp (-\Delta C / T)$, where $T$ is the temperature, if it would increase the cost by $\Delta(C)$. Also, in Figure 2, $s$ is the current schedule and $s^{\prime}$ is a neighboring schedule obtained from the current neighborhood space $\left(\mathcal{N}_{s}\right)$ by swapping two classes in time and/or space.

Thus the technique is essentially a generalization of the local optimization strategy, where, at non-zero temperatures, thermal excitations can facilitate escape from local minima.

1. Generate an initial schedule $s$.

2. Set the initial best schedule $s^{*}=s$.

3. Compute cost of $s: C(s)$.

4. Compute initial temperature $T_{0}$.

5. Set the temperature $T=T_{0}$.

6. While stop criterion is not satisfied do:

a) Repeat Markov chain length (M) times:

i. Select a random neighbor $s^{\prime}$ to the current schedule, $\left(s^{\prime} \subset \mathcal{N}_{s}\right)$.

ii. Set $\Delta(C)=C\left(s^{\prime}\right)-C(s)$.

iii. If $(\Delta(C) \leq 0$ \{downhill move $\})$ :

- Set $s=s^{\prime}$.

- If $C(s)<C\left(s^{*}\right)$ then set $s^{*}=s$.

iv. If $(\Delta(C)>0$ \{uphill move $\})$ :

- Choose a random number $r$ uniformly from $[0,1]$.

- If $r<e^{-\Delta(C) / T}$ then set $s=s^{\prime}$.

b) Reduce (or update) temperature $T$.

7. Return the schedule $s^{*}$.

Fig. 2. The Simulated Annealing Algorithm

The SA algorithm has advantages and disadvantages compared to other global optimization techniques. Among its advantages are the relative ease of implementation, the applicability to almost any combinatorial optimization problem, the ability to provide reasonably good solutions for most problems (depending on the cooling schedule and update moves used), and the ease with which it can be combined with other heuristics, such as expert systems, forming quite useful hybrid methods for tackling a range of complex problems. SA is a robust technique, however, it does have some drawbacks. To obtain good results the update moves and the various tunable parameters used (such as the cooling rate) need to be carefully chosen, the runs often require a great deal of computer time, and many runs may be required. 
Depending on the problem to which it is applied, SA appears competitive with many of the best heuristics, as shown in the work of Johnson et al. [21].

\subsection{Timetabling Using the Annealing Algorithm}

The most obvious mapping of the timetabling problem into the SA algorithm involves the following constructs:

1. a state is a timetable containing the following sets:

- P: a set of professors.

- $C$ : a set of classes.

- $S$ : a set of students.

- R: a set of classrooms.

$-I$ : a set of time intervals.

2. a cost or "energy" $\mathrm{E}(P, C, S, R, I)$ such that:

- $\mathrm{E}(P)$ : is the cost of assigning more than maximum number of allowed classes $M_{p}$ to the same professor, plus scheduling one or more classes that cause a conflict in the professor's schedule.

- $\mathrm{E}(C)$ : is the cost of scheduling certain classes at/within the same time period in violation of the exclusion constraint, for example.

- $\mathrm{E}(S)$ : is the cost of having two or more classes conflict in time; plus cost of having in the schedule one or more classes that really don't meet the student's major, class requested, or class requirements; plus the cost of not having the classes evenly spread out over the week, etc.

- $\mathrm{E}(R)$ : is the cost resulting from assigning room(s) of the wrong size and/or type to a certain class.

- $\mathrm{E}(I)$ : is the cost of having more or less time periods than required, plus cost of an imbalanced class assignments (a certain period will have more classes assigned to than others, etc.).

3. A swap (or a move) is the exchange of one or more of the following: class $c_{i}$ with class $c_{j}$ in the set $C$ with respect to time periods $I_{i}$ and $I_{j}$, and/or with respect to classroom $R_{i}$ and $R_{j}$, respectively. Generally, this step is referred to as class swapping.

Along with all of the necessary constraints, the simulated annealing algorithm also takes as input data the following: the preprocessor output in the form of lists of scheduled and non-scheduled classes and their associated professors and room types, a list of rooms provided by the registrar's office, a department to building distance matrix, a list of students and their class preferences, and a list of classes that are not allowed to be scheduled simultaneously.

To use simulated annealing effectively, it is crucial to use a good cooling schedule, and a good method for choosing new trial schedules, in order to efficiently sample the search space. We have experimented with both these areas, which are discussed in the following sections. 


\subsection{The Annealing Schedules}

Three annealing schedules have been used in our experiments to update the temperature of the SA algorithm in Figure 2: geometric cooling, adaptive cooling, and adaptive reheating as a function of cost.

The first schedule we have used is geometric cooling, where the new temperature $\left(T^{\prime}\right)$ of the SA algorithm is computed using

$$
T^{\prime}=\alpha T,
$$

where $\alpha(0<\alpha<1)$ denotes the cooling factor. Typically the value of $\alpha$ is chosen in the range 0.90 to 0.99 . This cooling schedule has the advantage of being well understood, having a solid theoretical foundation, and being the most widely used annealing schedule. Our results obtained from using this standard cooling schedule will be used as a baseline for comparison with those using the other two schedules, which allow the rate of cooling to be varied.

The second annealing schedule we used is the method of reheating as a function of cost (RFC), which was used for timetabling by Abramson et al. [3], but the ideas behind it are due to Kirkpatrick et al. [22,23] and White [36]. Before introducing this schedule we first summarize a few relevant points on the concept of specific heat $\left(C_{H}\right)$. Specific heat is a measure of the variance of the cost (or energy) values of states at a given temperature. The higher the variance, the longer it presumably takes to reach equilibrium, and so the longer one should spend at the temperature, or alternatively, the slower one should lower the temperature.

Generally, in combinatorial optimization problems, phase transitions $[16,26]$ can be observed as sub-parts of the problem are resolved. In some of the work dealing with the traveling salesman problem using annealing [24], the authors often observe that the resolution of the overall structure of the solution occurs at high temperatures, and at low temperatures the fine details of the solution are resolved. As reported in [3], applying a reheating type procedure, depending on the phase, would allow the algorithm to spend more time in the low temperature phases, thus reducing the total amount of time required to solve a given problem.

In order to calculate the temperature at which a phase transition occurs, it is necessary to compute the specific heat of the system. A phase transition occurs at a temperature $T\left(C_{H}^{\max }\right)$ when the specific heat is maximal $\left(C_{H}^{\max }\right)$, and this triggers the change in the state ordering. If the best solution found to date has a high energy or cost then the super-structure may require re-arrangement. This can be done by raising the temperature to a level which is higher than the phase transition temperature $T\left(C_{H}^{\max }\right)$. Generally, the higher the current best cost, the higher the temperature which is required to escape the local minimum. To compute the aforementioned maximum specific heat, we employ the following steps $[3,34,27]$.

At each temperature $T$, the annealing algorithm generates a set of configurations $\mathcal{C}(T)$. Let $C_{i}$ denote the cost of configuration $i, C(T)$ is the average cost at temperature $T$, and $\sigma(T)$ is the standard deviation of the cost at $T$. 
At temperature $T$, the probability distribution for configurations is:

$$
P_{i}(T)=\frac{e^{\frac{-C_{i}}{k T}}}{\sum_{j} e^{\frac{-C_{j}}{k T}}} .
$$

The average cost is computed as:

$$
<C(T)>=\sum_{i \in \mathcal{C}} C_{i} P_{i}(T) .
$$

Therefore, the average square cost is:

$$
<C^{2}(T)>=\sum_{i \in \mathcal{C}} C_{i}^{2} P_{i}(T) .
$$

The variance of the cost is:

$$
\sigma^{2}(T)=<C^{2}(T)>-<C(T)>^{2} .
$$

Now, the specific heat is defined as:

$$
C_{H}(T)=\frac{\sigma^{2}(T)}{T^{2}} .
$$

The temperature $T\left(C_{H}^{\max }\right)$ at which the maximum specific heat occurs, or at which the system undergoes a phase transition, can thus be found.

Reheating sets the new temperature to be

$$
T=K \cdot C_{b}+T\left(C_{H}^{\max }\right)
$$

where $K$ is a tunable parameter and $C_{b}$ is the current best cost. Reheating is done when the temperature drops below the phase transition (the point of maximum specific heat) and there has been no decrease in cost for a specified number of iterations, i.e. the system gets stuck in a local minimum. Reheating increases the temperature above the phase transition (see equation 11), in order to produce enough of a change in the configuration to allow it to explore other minima when the temperature is reduced again.

The third cooling schedule we have tried is adaptive cooling. In this case, a new temperature is computed based on the specific heat, i.e. the standard deviation of all costs obtained at the current $T$. The idea here is to keep the system close to equilibrium, by cooling slower close to the phase transition, where the specific heat is large. There are many different ways of implementing this idea, we have chosen the approach taken by Huang et al. [18], which was shown to yield an efficient cooling schedule. Let $T_{j}$ denote the current temperature, at step $j$ of the annealing schedule. After calculating $\sigma\left(T_{j}\right)$ from equation 9 , the new temperature $T_{j+1}$ is computed as follows:

$$
T_{j+1}=T_{j} \cdot e^{-\frac{a T_{j}}{\bar{\sigma}\left(T_{j}\right)}}
$$


where $a$ is a tunable parameter. Following suggestions by Otten and van Ginneken [27] and Diekmann et al. [6], $\sigma\left(T_{j}\right)$ is smoothed out in order to avoid any dependencies of the temperature decrement on large changes in the standard deviation $\sigma$. We used the following standard method to provide a smoothed standard deviation $\bar{\sigma}$ :

$$
\bar{\sigma}\left(T_{j+1}\right)=(1-\omega) \sigma\left(T_{j+1}\right)+\omega \sigma\left(T_{j}\right) \frac{T_{j+1}}{T_{j}}
$$

and set $\omega$ to 0.95 . This smoothing function is used because it follows (from the form of the Boltzmann distribution, see $[32,36])$ that it preserves the key relationship:

$$
\frac{d}{d T} C(T)=\frac{\bar{\sigma}^{2}(T)}{T^{2}}=C_{H}
$$

Note that reheating can be used in conjunction with any cooling schedule. We have used it with adaptive cooling.

\subsection{The Choice of Moves}

The performance of any application of simulated annealing is highly dependent on the method used to select a new trial configuration of the system for the Metropolis update. In order for the annealing algorithm to work well, it must be able to effectively sample the parameter space, which can only be done with efficient moves.

The simplest method for choosing a move is to swap the rooms or timeslots of two randomly selected classes. However this is extremely inefficient, since most of the time random swapping of classes will increase the overall cost, especially if we are already close to obtaining a valid solution (i.e. at low temperature), and will likely be rejected in the Metropolis procedure. This low acceptance of the moves means this simple method is very inefficient, since a lot of computation is required to compute the change in cost and do the Metropolis step, only to reject the move.

What is needed is a strategy for choosing moves that are more likely to be accepted. A simple example is in the choice of room. If we randomly choose a new room from the list of all rooms, it will most likely be rejected, since it may be too small for the class, or an auditorium when, for example, a laboratory is needed. One possibility is to create a subset of all the rooms which fulfill the hard constraints on the room for that particular class, such as the size and type of room. Now we just make a random selection for a room for that class only from this subset of feasible rooms, with an acceptance probability that is sure to be much higher. In addition, each class in our data set comes with a "type-ofspace-needed" tag which is used along with other information to assign the class to the right room. This effectively separates the updates into independent sets based on room type, so for example, laboratories are scheduled separately from lectures. In our method we carry out the scheduling of lectures first, followed by scheduling of laboratories making sure that during the course of this process no lecture and its associated laboratory are scheduled in the same time period. 
In effect, we have embedded a simple expert system into the annealing algorithm in order to improve the choice of moves, as well as using a more complex expert system as a preprocessor for the annealing step. When used to choose the moves for annealing, the main function of the rule-based system is to ensure that all the trial moves satisfy the hard constraints. Many of the rules dealing with the medium and soft constraints are softened or eliminated, since reducing the cost of these constraints is done using the Metropolis update in the annealing algorithm.

Another of the modifications to the rule-based system is that while the version used in the preprocessor is completely deterministic, the version used in choosing the moves for annealing selects at random from multiple possibilities that satisfy the rules equally well. This extra freedom in choosing new schedules, plus the extra degree of randomness inherent in the annealing update, helps prevent the system from getting trapped in a local minimum before it can reach a valid schedule, which is the problem with the standard deterministic rule-based system.

To improve further on the move strategy, we can take the subset of possible move choices that we have created for each class, and choose from them probabilistically rather than randomly. There may be certain kinds of moves that are more likely to be effective, so our move strategy is to select these moves with a higher probability. For example, swapping a higher level class (e.g. graduate) with a lower level class (e.g. a first or a second year type) generally has a higher acceptance, since there is little overlap between students taking these classes. Furthermore, we have experimented with two kinds of swaps, those that only involve classes offered by the same department or college and the second, swaps between classes of different departments and colleges.

Generally, the swap methods we have taken here can be considered as heuristics for pruning the neighborhood or narrowing the search space, which provides much more efficient moves and in turn an overall improvement in the results.

\section{Experimental Results}

Our computations were done with a number of goals in mind. The main objective was to provide a schedule which satisfied all hard constraints and minimized the cost of medium and soft constraints, using real-life data sets for a large university. We also aimed to find an acceptable set of annealing parameters and move strategies for general timetabling problems of this kind, and to study the effect of using a preprocessor to provide the annealing program with a good starting point. Finally, we wanted to make a comparison of the performance of the three different cooling schedules, geometric cooling, adaptive cooling, and reheating based on cost.

We spent quite some time finding optimal values for the various parameters for the annealing schedule, such as the initial temperature, the parameters controlling the rate of cooling ( $\alpha$ for geometric cooling, $a$ for adaptive cooling) and reheating $(K)$, and the number of iterations at each temperature (for more de- 
tails, see Ref. [11]). Johnson et al. [21] noted in their SA implementation for the traveling salesman problem (TSP) that the number of steps at each temperature (or the size of the Markov chain) needed to be at least proportional to the "neighborhood" size in order to maintain a high-quality result. From our experiments we found the same to be true for the scheduling problem, even though it is very different from the TSP. Furthermore, in a few tests for one semester we fixed the number of classes and professors but varied the number of rooms and time slots, and found that the final result improves as the number of iterations in the Markov chain becomes proportional to a combination of the number of classes, rooms and time slots. We also observed the same behavior when we fixed the number of rooms and time slots but varied number of classes.

Our study case involved real scheduling data covering three semesters at Syracuse University. The size and type of the three-semester data is shown in Table 1. Nine types of rooms were used: auditoriums, classrooms, computer clusters, conference rooms, seminar rooms, studios, laboratories, theaters, and unspecified types. Staff and teaching assistants are considered part of the set of professors. Third semester (summer) data was much smaller than other semesters, however, there were additional space and time constraints and fewer available rooms. Our data was quite large in comparison to data used by other researchers. For example, high school data used by Peterson and colleagues [13,14] consists of approximately 1000 students, 20 different possible majors, and an overall periodic school schedule (over weeks). In the case of Abramson et al. [2], their data set was created randomly and was relatively small, and they stated that problems involving more than 300 tuples were very difficult to solve.

Table 1 lists all major components of the data we have used. Timetabling problems can be characterized by their sparseness. After the required number of lessons $N_{l}$ have been scheduled, there will be $N_{s p}=\left(N_{x} N_{t}-N_{l}\right)$ spare spacetime slots, hence, the sparseness ratio of the problem is defined as the ratio $N_{s p} /\left(N_{x} N_{t}\right)$. The denser the problem, the lower the sparseness ratio, and the harder the problem is to solve. Also, for dense problems, there is an additional correlation involving the problem size. Table 2 shows the sparseness of the threesemester data. For university scheduling, the sparseness ratio generally decreases as the data size (particularly the number of classes) increases, so the problem becomes harder to solve. Including student preferences makes the problem much harder, but these are viewed as medium constraints and thus are not necessarily satisfied in a valid solution.

Our overall results are shown in Tables 3 and 4 . These tables show the percentage of classes that could be scheduled in accordance with the hard constraints. In each case (apart from the expert system, which is purely deterministic), we have done 10 runs (with the same parameters, just different random numbers), and the tables show the average of the 10 runs, as well as the best and worst results. The MFA results are different only due to having different initial conditions. Each simulated annealing run takes about 10 to 20 hours on a Unix workstation, while a single MFA run takes approximately an hour and an expert system run takes close to two hours. 
Table 1. Size of the data set for each of the three semesters.

\begin{tabular}{|l||c|c|c||}
\hline & First Semester & Second Semester & Third Semester \\
\hline \hline Rooms & 509 & 509 & 120 \\
\hline Classes & 3839 & 3590 & 687 \\
\hline Professors & 1190 & 1200 & 334 \\
\hline Students & 13653 & 13653 & 2600 \\
\hline Buildings & 43 & 43 & 11 \\
\hline Schools and/or Colleges & 20 & 21 & 17 \\
\hline $\begin{array}{l}\text { Departments or } \\
\text { Course Prefixes }\end{array}$ & 143 & 141 & 108 \\
\hline Areas of Study (majors) & 200 & 200 & 200 \\
\hline
\end{tabular}

Table 2. The sparseness ratios of the problem for the data sets for each of the three semesters. Lower values indicate a harder problem.

\begin{tabular}{|l||c|}
\hline $\begin{array}{l}\text { Academic } \\
\text { Time Period }\end{array}$ & $\begin{array}{c}\text { Sparseness } \\
\text { ratio }\end{array}$ \\
\hline \hline First Semester & 0.50 \\
\hline Second Semester & 0.53 \\
\hline Third Semester & 0.62 \\
\hline
\end{tabular}

Table 3. Percentage of classes scheduled using the different methods. The averages and highest and lowest values were obtained using 10 independent runs for simulated annealing (SA) and mean-field annealing (MFA). The expert system (ES) is deterministic so the results are from a single run. No preprocessor was used with the three methods.

\begin{tabular}{|l|l||c|c|c||}
\hline $\begin{array}{l}\text { Academic } \\
\text { Time Period }\end{array}$ & Algorithm & $\begin{array}{c}\text { Scheduled } \\
(\text { average) } \\
\%\end{array}$ & $\begin{array}{c}\text { Highest } \\
\text { Scheduled } \\
\%\end{array}$ & $\begin{array}{c}\text { Lowest } \\
\text { Scheduled } \\
\%\end{array}$ \\
\hline \hline First Semester & SA (geometric) & 65.00 & 67.50 & 56.80 \\
& SA (adaptive) & 67.80 & 70.15 & 61.20 \\
& SA (cost-based) & 70.20 & 72.28 & 68.80 \\
& ES & 76.65 & 76.65 & 76.65 \\
& MFA & 65.60 & 71.00 & 61.00 \\
\hline Second Semester & SA (geometric) & 65.65 & 68.00 & 57.10 \\
& SA (adaptive) & 68.50 & 70.10 & 60.77 \\
& SA (cost-based) & 75.14 & 77.68 & 70.82 \\
& ES & 79.00 & 79.00 & 79.00 \\
& MFA & 67.20 & 75.00 & 65.00 \\
\hline Third Semester & SA (geometric) & 83.10 & 86.44 & 68.50 \\
& SA (adaptive) & 85.80 & 89.00 & 70.75 \\
& SA (cost-based) & 91.20 & 95.18 & 85.00 \\
& ES & 96.80 & 96.80 & 96.80 \\
& MFA & 88.00 & 95.00 & 82.00 \\
\hline
\end{tabular}


Table 4. Percentage of scheduled classes, averaged over 10 runs of the same initial temperature and other parameters, for three terms using simulated annealing with an expert system as preprocessor.

\begin{tabular}{|l|l|c|c|c||}
\hline $\begin{array}{l}\text { Academic } \\
\text { Time Period }\end{array}$ & Algorithm & $\begin{array}{c}\text { Scheduled } \\
\text { (average) } \\
\%\end{array}$ & $\begin{array}{c}\text { Highest } \\
\text { Scheduled } \\
\%\end{array}$ & $\begin{array}{c}\text { Lowest } \\
\text { Scheduled } \\
\%\end{array}$ \\
\hline \hline First Semester & SA (geometric) & 93.90 & 95.12 & 85.20 \\
& SA (adaptive) & 98.80 & 99.20 & 95.00 \\
& SA (cost-based) & 100.0 & 100.0 & 100.0 \\
\hline Second Semester & SA (geometric) & 95.00 & 98.95 & 89.40 \\
& SA (adaptive) & 99.00 & 99.50 & 98.50 \\
& SA (cost-based) & 100.0 & 100.0 & 100.0 \\
\hline Third Semester & SA (geometric) & 97.60 & 98.88 & 90.90 \\
& SA (adaptive) & 100.0 & 100.0 & 100.0 \\
& SA (cost-based) & 100.0 & 100.0 & 100.0 \\
\hline
\end{tabular}

As expected, each of the methods did much better for the third (summer) semester data, which has a higher sparseness ratio. Our results also confirm what we expected for the different cooling schedules for simulated annealing, in that adaptive cooling performs better than geometric cooling, and reheating improves the result even further.

When a random initial configuration is used, simulated annealing performs very poorly, even worse than the expert system (ES). However, there is a dramatic improvement in performance when a preprocessor is used to provide a good starting point for the annealing. In that case, using the best cooling schedule of adaptive cooling with reheating as a function of cost, we are able to find a valid class schedule every time.

In the case of mean-field annealing, the overall results are generally below those of SA and ES. In addition, we have found in the implementation of this method that the results were quite sensitive to the size of the data as well the type of constraints involved. If we confine ourselves to the set of hard constraints, the results are as good as or even better than the other methods. However if we take into account the medium and soft constraints, that is, the overall cost function, this method does not perform as well.

Student preferences are included only as medium constraints in our implementation, meaning that these do not have to be satisfied for a valid solution, but they have a high priority. For the valid schedules we have produced, approximately $75 \%$ of the student preferences were satisfied. This is reasonably good (particularly since other approaches do not deal with student preferences at all), but we are working to improve upon this result. 


\section{Conclusions}

We have successfully applied simulated annealing to the difficult problem of academic scheduling for a large university. Feasible schedules were obtained for real data sets, including student preferences, without requiring enormous computational effort.

Mean-field annealing works well for small scheduling problems, but does not appear to scale well to large problems with many complex constraints. For this problem, both simulated annealing and the rule-based system were more effective than MFA. It is more difficult to tune the parameters for MFA than for simulated annealing, and because of the complexity and size of the Potts neural encoding, there seems to be no clear way of preserving the state of a good initial configuration provided by a preprocessor when using MFA.

Using a preprocessor to provide a good initial state greatly improved the quality of the results for simulated annealing. In theory, using a good initial state should not be necessary, and any initial state should give a good result, however in practice, we do not have an ideal cooling schedule for annealing, or an ideal method for choosing trial moves and efficiently exploring the search space, and there are restrictions on how long the simulation can take. In general, for very hard problems with large parameter spaces that can be difficult to search efficiently, and for which very slow cooling would be much too time-consuming, we might expect that a good initial solution would be helpful. We used a fairly complex rule-based expert system for the preprocessor, however the type of preprocessor may not be crucial. Other fast heuristics could possibly be used, for example a graph coloring approach [25], or it may be possible to just utilize the schedule from the same semester for the previous year. A modified version of the rule-based system was used to choose the trial moves for the simulated annealing, and the high acceptance rate provided by this system was crucial to obtaining good results.

As expected, for the simulated annealing, adaptive cooling performed better than geometric cooling, and using reheating improved the results even further. The best results were obtained using simulated annealing with adaptive cooling and reheating as a function of cost, and with a rule-based preprocessor to provide a good initial solution. Using this method, and with careful selection of parameters and update steps, we were able to generate solutions to the class scheduling problem using real data for a large university. None of the other methods were able to provide a complete solution.

Our main conclusion from this work is that simulated annealing, with a good cooling schedule, optimized parameters, carefully selected update moves, and a good initial solution provided by a preprocessor, can be used to solve the academic scheduling problem at a large university, including student preferences. Similar approaches should prove fruitful for other difficult scheduling problems. 


\section{Acknowledgments}

The first author is very grateful for the valuable discussion and help of Robert Irwin in converting and formatting the registration data prior to the scheduling process. We also would like to thank Andrew Gee and Martin Simmen for the useful comments and suggestions, and Carsten Peterson for the pointers and comments about his papers. Many thanks go to Karen Bedard for providing us with the data and answering so many questions we had about it, Meg Cortese for providing us with a set of building constraints for various departments, and Prof. Ben Ware, Vice President for Research and Computing at Syracuse University, for his support and encouragement.

\section{References}

1. Aarts, E. H., J. Korst, and P. J. van Laarhoven, "Simulated annealing," in Local Search in Combinatorial Optimization, E. H. Aarts and J. K. Lenstra (eds.), John Wiley and Sons, 1997.

2. Abramson, D., "Constructing school timetables using simulated annealing: sequential and parallel algorithms," Management Science 37(1), 98-113, 1991.

3. Abramson, D., H. Dang, and M. Krishnamoorthy, "An Empirical Study of Simulated Annealing Cooling Schedules," Griffith Univ. report, Nathan, Qld, Aus. 1994; "Simulated Annealing Cooling Schedules for the School Timetabling Problem," submitted to Asia Pacific Journal of Operations Research, 1996.

4. Burke, E., and P. Ross, eds., Practice and Theory of Automated Timetabling, First International Conference, Edinburgh, 1995 : Selected Papers, Lecture Notes in Computer Science no. 1153, Springer, New York, 1996.

5. de Werra, D., "An introduction to timetabling," European Journal of Operational Research 19, 151-162, 1985.

6. Diekmann, R., R. Lüling, and J. Simon, "Problem independent distributed simulated annealing and its applications," in Applied Simulated Annealing, R. V. Vidal ed., Lecture Notes in Economics and Mathematical Systems, no. 396, SpringerVerlag, 1993.

7. Dowsland, K., "Using Simulated Annealing for Efficient Allocation of Students to Practical Classes", Working Paper, Statistics and OR Group, European Business Management School, University College of Swansea, UK, 1994.

8. Dowsland, K. and J. Thompson, "Variants of Simulated Annealing for the Examination Timetabling Problem," Working Paper, Statistics and OR Group, European Business Management School, University College of Swansea, UK, 1994.

9. Eiselt H. A., and G. Laporte, "Combinatorial Optimization Problems with Soft and Hard Requirements," J. Operational Research Society, vol. 38, No. 9, pp. 785-795, 1987.

10. Elmohamed, S., G. C. Fox, P. Coddington, "Course Scheduling using Mean-Field Annealing, Part I: algorithm and Part II: implementation," Northeast Parallel Architectures Center technical report SCCS-782, Syracuse University, Syracuse, NY, 1996.

11. Elmohamed, S., P. Coddington, G.C. Fox, "Academic Scheduling using Simulated Annealing with a Rule-Based Preprocessor", Northeast Parallel Architectures Center technical report SCCS-781, Syracuse University, Syracuse, NY, 1997. 
12. Gee, Andrew, private communication.

13. Gislén, L., B. Söderberg, C. Peterson, "Teachers and Classes with Neural Nets," International Journal of Neural Systems 1, 167 (1989).

14. Gislén, L., B. Söderberg, C. Peterson, "Complex scheduling with Potts neural networks," Neural Computation, 4, 805-831, 1992.

15. Hertz, J., A. Krogh and R. Palmer, Introduction to the Theory of Neural Computation, Addison-Wesley, Redwood City, CA, 1991.

16. Hogg, T., B. Huberman, and C. Williams (editors), Artificial Intelligence, special issue on Phase transitions and the search space, p. 81, 1996.

17. Hopfield, J. J., and D. W. Tank, "Neural Computation of Decisions in Optimization Problems," Biological Cybernetics 52, 141 (1985).

18. Huang, M., F. Romeo, and A. Sangiovanni-Vincentelli, "An efficient general cooling schedule for simulated annealing," Proc. of the IEEE International Conference on Computer Aided Design (ICCAD), pp. 381-384, 1986.

19. Johnson, D., C. Aragon, L. McGeoch, and C. Schevon, "Optimization by Simulated Annealing: an Experimental Evaluation, Part I (Graph Partitioning)," Operations Research 37, 865-892 (1989).

20. Johnson, D., C. Aragon, L. McGeoch, and C. Schevon, "Optimization by Simulated Annealing: an Experimental Evaluation, Part II (Graph Coloring and number partitioning)," Operations Research 39, No. 3, 865-892 (1991).

21. Johnson, D., and L. McGeoch, "The Traveling Salesman Problem: A Case Study in Local Optimization," in Local Search in Combinatorial Optimization, E. H. Aarts and J. K. Lenstra (eds.), Wiley and Sons, 1997.

22. Kirkpatrick, S., C. D. Gelatt, Jr., and M. P. Vecchi, "Optimization by Simulated Annealing," Science 220, 671-680, (13 May 1983).

23. Kirkpatrick, S., "Optimization by simulated annealing: Quantitative studies," J. Stat. Physics 34, 976-986 (1984).

24. Lister, R.," Annealing Networks and Fractal Landscapes," Proc. IEEE International Conference on Neural Nets, March 1993, Vol. I, pp 257-262.

25. Miner, S., S. Elmohamed, and H. W. Yau, "Optimizing Timetabling Solutions Using Graph Coloring," 1995 NPAC REU program, NPAC, Syracuse University, Syracuse, NY, 1995.

26. Mouritsen, O. G., Computer Studies of Phase Transitions and Critical Phenomena, Springer-Verlag, Berlin, 1984.

27. Otten, R., and L. van Ginneken, The Annealing Algorithm, Kluwer Academic Publishers, 1989.

28. Peterson, C., and B. Söderberg, "Artificial Neural Networks and Combinatorial Optimization Problems," Local Search in Combinatorial Optimization, E.H.L. Aarts and J.K. Lenstra (eds.), Wiley and Sons, 1997.

29. Peterson, C., and B. Söderberg, "A New Method for Mapping Optimization Problems onto Neural Nets", International Journal of Neural Systems 1, 3 (1989).

30. Schaerf, A., "A survey of automated timetabling," Department of Software Technology, Report CS-R9567, CWI, Amsterdam, The Netherlands.

31. Simmen, Martin, Personal Communication.

32. Sorkin, G., Theory and Practice of Simulated Annealing on Special Energy Landscapes, PhD. Thesis, Dept. of Electrical Engineering and Computer Science, University of California, Berkeley, July 1991.

33. Thompson, J., and K. Dowsland, "General Cooling Schedules for Simulated Annealing Based Timetabling Systems," Proceedings of the 1st International Conf. on the Practice and Theory of Automated Timetabling, Napier Univ., Edinburgh 1995. 
112 M.A.S. Elmohamed, P. Coddington, and G. Fox

34. van Laarhoven, P. J. and E. H. Aarts, Simulated Annealing: Theory and Applications. D. Reidel, Dordrecht, 1987.

35. Vidal, R. V. ed., Applied Simulated Annealing, Lecture Notes in Economics and Mathematical Systems no. 396, Springer-Verlag, 1993.

36. White, S. R., "Concepts of scale in simulated annealing," Proceedings of the IEEE International Conference on Circuit Design, pp 646-651, 1984. 